\title{
Ausência de variação da flexibilidade durante o ciclo menstrual em universitárias*
}

\author{
Christianne Pereira Giesbrecht Chaves ${ }^{1}$, Roberto Simão ${ }^{1}$ e Claudio Gil Soares de Araújo ${ }^{1,2}$
}

\section{RESUMO}

Fundamentação: Algumas variáveis da aptidão física são marcadamente influenciadas pelas fases do ciclo menstrual (CM); contudo, há pouca informação sobre eventuais modificações na flexibilidade. Objetivo: Analisar o comportamento da flexibilidade corporal - global, por articulação e por movimento - em mulheres adultas jovens nas diferentes fases do CM. Métodos: Estudou-se uma amostra de 15 mulheres. O grupo experimental (GE) foi constituído de alunas eumenorréicas, enquanto o grupo controle (GC) incluía alunas em uso regular havia pelo menos um mês de anticoncepcional oral (AO). Dados referentes ao período menstrual e ao uso de AO foram obtidos através da aplicação de um questionário. A flexibilidade foi avaliada pelo Flexiteste, permitindo uma análise da flexibilidade global (Flexíndice), por articulação, por movimento e em sua variabilidade. Os Flexíndices foram ainda comparados com os correspondentes percentis para gênero e idade. Para a análise estatística das três medidas do GE - fases folicular, ovulatória e lútea - e das duas do GC - entre dias cinco e 26 do ciclo, utilizou-se ANOVA ou teste $t$ conforme apropriado. Resultados: Não houve diferenças na flexibilidade, movimento a movimento ou Flexíndice nas diferentes fases do CM no GE e nas duas medidas do GC ( $p>0,05)$. O percentil do Flexíndice para o GC variou de 14 a 35 e no $\mathrm{GE}$, de 14 a 80 . Analisando as articulações e os índices de variabilidade, não foram encontradas diferenças significativas com as fases do $\mathrm{CM}(\mathrm{p}>0,05)$, com a exceção do índice de variabilidade distal-proximal entre as fases ovu-

\footnotetext{
* Programa de Pós-Graduação em Educação Física da Universidade Gama Filho e Clinimex - Clínica de Medicina do Exercício, Rio de Janeiro, RJ.

1. Programa de Pós-Graduação em Educação Física da Universidade Gama Filho.

2. Clinimex, Clínica de Medicina do Exercício.

Recebido em 23/5/02

Aceito em 14/9/02
}

Endereço para correspondência:

Dr. Claudio Gil S. Araújo - Clínica de Medicina do Exercício (Clinimex)

Rua Siqueira Campos, 93/101

22031-070 - Rio de Janeiro, RJ, Brasil

E-mail: cgaraujo@iis.com.br latória e folicular no GE. Conclusão: Os dados obtidos não corroboram a impressão empírica de que a flexibilidade varia durante as fases do CM. Não se pode, contudo, afastar a hipótese de que a ausência de variações tenha ocorrido devido a alguma característica da amostra ou por limitações do Flexiteste em identificar mínimas variações, sendo necessária a realização de outros estudos.

Palavras-chave: Flexibilidade. Ciclo menstrual. Anovulatório oral.

\section{ABSTRACT \\ Lack of flexibility variation during menstrual cycle in university students}

Background: Some physical components are markedly influenced by the different phases of menstrual cycle $(M C)$; however, there is little information about changes in flexibility. Objectives: To analyze the behavior of corporal flexibility - global, per joints and per movements - in adult young women during different phases of the MC. Methods: A sample of 15 women was studied. The experimental group (EG) included students with regular $M C$, and the control group $(C G)$ had students that had been taking oral contraceptives $(O C)$ for at least one month. Data concerning the $M C$ and $O C$ use were acquired by questionnaire. The flexibility was evaluated by Flexitest, allowing a global analysis of flexibility (Flexindex), per joints, per movements and its variability. The Flexindices were compared with corresponding percentiles as to gender and age. For the statistical analysis in three phases of the EG-follicular, ovulatory and luteal - and the CG in two different moments between the days 5 and 26 of the MC, ANOVA and T-test were used as appropriate. Results: There were no significant differences in flexibility, per movements or Flexindex during the phases of $M C$ in the EG or in the $C G(p>0.05)$. The Flexindex percentiles ranged from 14 to 35 for the $C G$, and from 14 to 80 for the EG. No significant differences between the joints and the variability indices during the $M C$ were observed ( $p>0.05$ ), except for the distal-proximal variability indices between the follicular and ovulatory phases on the EG. Conclusion: These data do not corroborate the empirical impression that flexibility changes 
during MC phases. However, it is possible that the lack of variation could have occurred due to some sample characteristics or to Flexitest limitations in identifying small changes, which poses the need for other studies.

Key words: Flexibility. Menstrual cycle. Oral contraceptive.

\section{INTRODUÇÃO}

O ciclo menstrual (CM) dura, em média, 28 dias, podendo ser dividido em três fases distintas: folicular, ovulatória e lútea. A fase folicular inicia-se no primeiro dia da menstruação e dura entre nove e 23 dias; a fase ovulatória pode durar até três dias e a fase lútea vai do fim da ovulação até o início do fluxo menstrual ${ }^{1,2}$. Outros autores ${ }^{3,4}$ levam em conta apenas as fases folicular e lútea, inserindo a fase ovulatória como uma etapa tardia dentro da fase folicular. Cada uma dessas fases caracteriza-se por um número específico de dias e por perfis específicos de concentrações séricas dos hormônios sexuais ${ }^{3}$.

$\mathrm{Na}$ fase folicular e ovulatória, grandes quantidades de estrogênio são secretadas, enquanto na fase lútea ocorre o predomínio de progesterona e, em menor grau, do estradiol. A fase lútea caracteriza-se pelo aumento acentuado dos níveis séricos de progesterona, alcançando o seu pico máximo logo após a ovulação e que são responsáveis pelo aumento da temperatura corporal basal observada nesta fase. Simultaneamente, o estrogênio sérico volta a aumentar nessa fase, após redução importante durante a ovulação. Nos últimos dias do CM, o corpo lúteo vai-se degenerando e os níveis de progesterona e estrogênio caem, provocando o início do fluxo menstrual e um novo ciclo se repete ${ }^{3}$.

Há estudos sugerindo que o desempenho físico pode variar consideravelmente durante as distintas fases do CM, muito provavelmente em função das variações de temperatura corporal e do metabolismo que acompanham as curvas hormonais ${ }^{2,5-7}$. Enquanto há dados relativos ao comportamento da potência aeróbia e da força muscular ${ }^{8-10}$, pouco se conhece em relação à flexibilidade. Relatos isolados de bailarinas sugerem que a mobilidade articular se modifica ao longo do CM. Considerando o perfil de curva dos níveis séricos dos hormônios femininos durante o $\mathrm{CM}$, especialmente a ação da progesterona sobre o aumento da temperatura corporal após a ovulação, poder-se-ia especular se a flexibilidade estaria aumentada na fase lútea.

O objetivo deste estudo foi analisar o comportamento da flexibilidade corporal - global, por articulação, por movimento e em sua variabilidade - em mulheres adultas jovens nas diferentes fases do CM.

\section{MATERIAIS E MÉTODOS}

Após um estudo piloto com oito universitárias, no qual se constatou a viabilidade da pesquisa, foram ajustados alguns aspectos operacionais e, a seguir, avaliadas 20 outras universitárias do curso de graduação em Educação Física na Universidade Gama Filho, do Rio de Janeiro. Dentre as alunas participantes da pesquisa, somente em 15 delas foi possível obter todas as medidas completas e ter, então, seus dados utilizados para posterior análise.

Essas alunas foram recrutadas a partir de convite formulado pela investigadora principal durante as aulas regulares. Especificamente, foram convidadas alunas que possuíssem ciclos menstruais regulares, que não estivessem grávidas e não tivessem a intenção de fazê-lo nos próximos meses. Os professores que ministravam as aulas estimularam a participação das alunas como atividade disciplinar complementar.

Antes de iniciar a coleta de dados, as alunas preencheram um termo de consentimento e responderam a um questionário com informações pertinentes ao $\mathrm{CM}$ e ao eventual uso de anticoncepcionais hormonais. Constituiu-se um grupo experimental (GE) com as alunas eumenorréicas, considerando-se como grupo controle (GC) as alunas que faziam uso regular, havia pelo menos um ciclo, de anticoncepcional hormonal. As alunas foram, assim, divididas em oito no $\mathrm{GC}$, com a idade variando de 19 a 25 anos $(22,3 \pm 1,3$ anos; média \pm desvio padrão) e sete no $\mathrm{GE}$, com idade compreendida entre 19 e 24 anos (21,0 $\pm 1,7$ anos).

A coleta de dados ocorreu no primeiro semestre letivo de 2001. As alunas foram medidas durante quatro semanas consecutivas, sempre no mesmo dia da semana (quinta-feira) e aproximadamente no mesmo horário (entre 8 e $10 \mathrm{~h}$ da manhã). Todas as medidas foram feitas sempre na mesma sala, com temperatura ambiente controlada entre 20 e 25 graus centígrados. Semanalmente, a investigadora questionava as alunas sobre a presença ou ausência de fluxo menstrual. As alunas estavam vestidas com roupas adequadas para a prática de exercícios, de forma que a execução dos movimentos não fosse prejudicada. Nenhuma aluna havia praticado qualquer exercício prévio ou estava havia pelo menos uma hora sem praticar qualquer exercício físico mais intenso (superior a seis METs).

O instrumento utilizado para a avaliação da flexibilidade foi o Flexiteste ${ }^{11,12}$, realizado por uma única avaliadora previamente treinada na sua utilização e sem conhecimento prévio da alocação de cada aluna no grupo, o que ficou sob controle de uma colaboradora, que anotava os resultados à medida que eles eram obtidos.

O Flexiteste consiste na medida e avaliação da amplitude fisiológica passiva máxima de 20 movimentos articula- 
res sempre feitos no dimídio direito e 36 se considerados bilateralmente (tabela 1). O Flexiteste engloba as articulações do tornozelo, joelho, quadril, tronco, punho, cotovelo e ombro. Oito movimentos são feitos nos membros inferiores, três no tronco e os nove restantes nos membros superiores, sem aquecimento prévio. A medida é feita através da execução lenta do movimento pelo avaliador até a obtenção da amplitude máxima no avaliado, para posteriormente compará-la com os mapas de avaliação. Em geral, o ponto máximo da amplitude de movimento é detectado pelo relato de desconforto local pelo avaliado e/ou pela grande resistência mecânica à continuação do movimento.

Cada um dos movimentos é medido em uma escala crescente e descontínua de números inteiros de 0 a 4 , perfazendo um total de cinco valores possíveis. A atribuição dos valores numéricos é determinada quando a amplitude alcançada é igual à existente no mapa de avaliação. No caso de a amplitude alcançada em um determinado movimento encontrar-se entre dois valores do mapa de avaliação, o menor valor prevalecerá, mesmo que a amplitude esteja mais próxima do valor mais alto. Não existem valores fracionários ou intermediários. O tempo de execução do teste é, em geral, entre três e cinco minutos, empregando uma sequiência modificada dos movimentos, na qual são minimizadas as mudanças de postura corporal.

Pela natureza da escala e pelo modo como foram propositadamente desenhados os mapas de avaliação, observa-

\begin{tabular}{c} 
TABELA 1 \\
Movimentos do Flexiteste \\
\hline
\end{tabular}

\begin{tabular}{cll} 
Item & Articulação & \multicolumn{1}{c}{ Movimento } \\
I & Tornozelo & Flexão (dorsal) \\
II & Tornozelo & Extensão (flexão plantar) \\
III & J oelho & Flexão \\
IV & J oelho & Extensão \\
V & Quadril & Flexão \\
VI & Quadril & Extensão \\
VII & Quadril & Adução \\
VIII & Quadril & Abdução \\
IX & Tronco & Flexão \\
X & Tronco & Extensão \\
XI & Tronco & Flexão lateral \\
XII & Punho & Flexão \\
XIII & Punho & Extensão \\
XIV & Cotovelo & Flexão \\
XV & Cotovelo & Extensão \\
XVI & Ombro & Adução posterior \\
XVII & Ombro & Extensão e adução posterior \\
XVIII & Ombro & Extensão posterior \\
XIX & Ombro & Rotação lateral \\
XX & Ombro & Rotação medial \\
\hline
\end{tabular}

se distribuição gaussiana para os dados, de forma que a tendência central para todos os movimentos é o valor 2; os valores 1 e 3 são menos freqüentes e os valores extremos, isto é, 0 e 4, tendem a ser raros, especialmente em adultos jovens.

A análise do Flexiteste pode e deve ser feita para cada um dos movimentos e/ou articulações, porém, a soma de seus valores é denominada Flexíndice, um índice global de flexibilidade ou mobilidade articular. Os resultados do Flexíndice também podem ser analisados em função dos valores esperados para uma população da mesma idade e gênero através das tabelas de percentis ${ }^{13}$. O mesmo Flexíndice pode ser obtido por diversas combinações de escores dos 20 movimentos do Flexiteste e em decorrência desta possibilidade. Araújo ${ }^{14}$ propôs cinco índices de variabilidade para esses resultados, tornando possível distinguir perfis de mobilidade articular mesmo quando dois indivíduos apresentam valores iguais de Flexíndice.

De modo sucinto, esses cinco índices são: a) intermovimentos (IVIM), representando o desvio padrão dos escores individuais dos 20 movimentos do Flexiteste; b) interarticulações (IVIA), refletindo a variabilidade entre as mobilidades médias obtidas nas diversas articulações testadas; c) flexão-extensão (IVFE) - comparando a mobilidade nos movimentos de flexão e extensão; d) entre-segmentos (IVES) - comparando as medidas de mobilidade dos segmentos inferior e superior do corpo e e) distal-proximal (IVDP) identificando eventuais discrepâncias entre a mobilidade passiva máxima das articulações distais e proximais dos membros $^{14}$.

Os anticoncepcionais utilizados pelo GC eram compostos por progestagênio e estrogênio em iguais concentrações em todos os comprimidos, variando apenas os fármacos e a dosagem, conforme é detalhado na tabela 2.

Para definição da fase em relação ao dia do ciclo e posterior análise dos dados no GE, utilizamos o critério proposto por Wojtys et al. ${ }^{2}$, assumindo que teríamos a fase folicular do primeiro dia do ciclo - o dia em que ocorre a menstruação - até o nono dia, a fase ovulatória entre os dias 10 e 14, o que em geral ocorre em mulheres eumenorréicas; para a fase lútea considerou-se da segunda metade do ciclo (dia 15) até o dia que antecede a menstruação,

TABELA 2

Composição dos anovulatórios

Progestagênio

$0,075 \mathrm{mg}$ de gestodeno

$2 \mathrm{mg}$ de acetato de ciproterona $0,15 \mathrm{mg}$ de desogestrel

\section{Estrogênio}

$0,02 \mathrm{mg}$ de etinilestradiol $0,035 \mathrm{mg}$ de etinilestradiol 0,03de etinilestradiol 


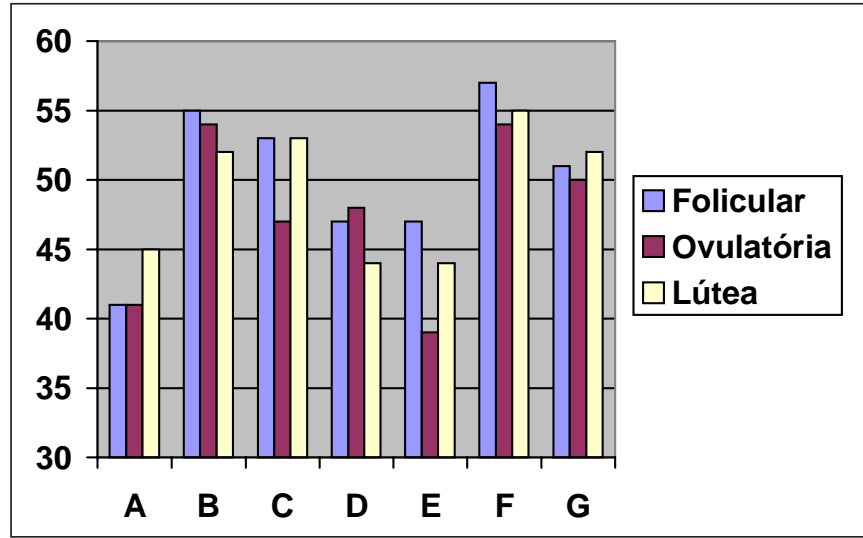

Fig. 1 - Flexíndice das universitárias do grupo experimental nas três medidas

independentemente da duração do ciclo individual. Para o GC foram utilizadas apenas duas medidas, compreendidas entre os dias 5 e 26 do ciclo, correspondendo a dias de uso regular do anovulatório hormonal, assumindo assim concentrações séricas hormonais idênticas nas duas medidas.

A análise estatística seguiu os métodos já preconizados e utilizados anteriormente em estudos com o Flexiteste ${ }^{13,14}$, incluindo estatística ANOVA para medidas repetidas para comparar os movimentos nas fases folicular, ovulatória e lútea no GE. No que concerne ao GC, em uso de anovulatório, foi utilizado o teste $t$ emparelhado, bicaudal, para comparar as duas medidas compreendidas entre os dias 7 e 26 do ciclo, que possuíam concentrações hormonais constantes. Eventuais comparações entre os dois grupos foram realizadas com teste $t$ bicaudal para amostras independentes. O nível de significância adotado para todos os procedimentos foi de $5 \%$.

\section{RESULTADOS}

Foi realizado um total de 740 medidas de mobilidade articular utilizando o Flexiteste, sendo 160 para dois momentos distintos no GC e 140 para cada uma das três fases, para o GE.

O Flexíndice para as fases folicular, lútea e ovulatória do GE foram, respectivamente, 50,1 [2,1] (média [erro padrão da média]), 49,3 [1,8] e 47,6 [2,2], e as duas do GC $47,0[0,9]$ e 47,1 [0,7] (figuras 1 e 2). Os resultados para a flexibilidade - por movimento, por articulação ou pelo Flexíndice - não diferiram durante as fases do $\mathrm{CM}$, seja nas duas medidas feitas com o GC (p > 0,05) (figura 1) ou nas três fases do ciclo nas alunas que não faziam uso de anovulatórios $(\mathrm{p}>0,05)$ (figura 2). Também não havia diferenças significativas no Flexíndice entre os GE e GC. Quanto aos resultados individuais do Flexíndice, eles não

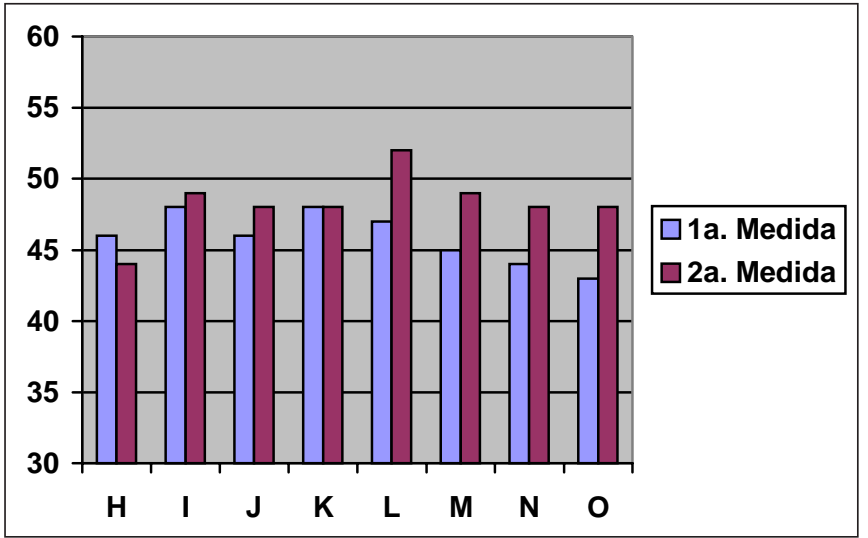

Fig. 2 - Flexíndice das universitárias do grupo controle nas duas medidas

variaram em apenas uma das alunas entre duas medidas, enquanto nas demais se observaram variações de até $20 \%$ sem uma relação objetiva com a fase do ciclo menstrual.

As universitárias testadas apresentavam níveis bastante variáveis de flexibilidade, correspondendo aos percentis 14 a 80 para o GE, utilizando os dados da fase lútea. O percentil para o GC variou de 14 a 35 , considerando as medidas obtidas entre o $17^{\circ}$ e o $23^{\circ}$ dia do ciclo. A mediana do percentil para o Flexíndice do GE ficou em 55 e a do GC em 32 quando comparada com os dados disponíveis para mesma idade e gênero (tabela 3 ).

No conjunto de medidas, a maior parte dos escores, como é esperado pela natureza da distribuição dos escores do Flexiteste, ficou no valor 2. Dentre as 740 medidas realiza-

\section{TABELA 3}

Percentis individuais do Flexíndice das universitárias do grupo controle (GC) e do grupo experimental (GE)$$
\text { GE }
$$

A

B

C

D

E

$\mathrm{F}$

G

\section{Percentis}

20

60

65

14

15

80

55

GC

$\mathrm{H} \quad 14$

I 35

J 32

K 25

L 35

M 14 
das, em 56 delas foi obtido um escore 4 e em apenas duas encontrou-se um escore 0 , um no movimento de tornozelo I na fase ovulatória e outro no ombro XIX na fase folicular, em duas meninas distintas do GE; em quase todas as universitárias encontrou-se pelo menos em um movimento um escore igual a 4.

Analisando movimento a movimento, observou-se que para o GE não houve qualquer variação na mobilidade para os movimentos de extensão do joelho e do cotovelo, nos quais todas as alunas apresentaram escore 2. Já para os movimentos I, II, VI, VII, XIX e XX, a maioria das alunas apresentou variação nas medidas de mobilidade articular ao longo das três fases do CM; em apenas dois únicos casos (duas alunas distintas), essa diferença chegou a dois pontos nos movimentos I e XIX. Em relação ao GC, em quatro dos movimentos não houve qualquer variação das medidas - III, IV, IX e XVIII -, interessantemente, movimentos distintos daqueles em que houve ausência de mudanças no GE. Já para os movimentos VI, VIII, X e XVI, verificaramse mudanças na maioria das alunas. Tal como ocorreu no GE, apenas em um único caso, uma aluna do GC teve uma diferença entre as medidas que alcançou dois pontos no movimento XIX.

Em relação aos índices de variabilidade, observou-se que praticamente $2 / 3$ dos escores se encontravam dentro da faixa de normalidade, percentual que foi bastante semelhante nos dois grupos $(p>0,05)$. Apenas no que se refere ao índice de variabilidade distal-proximal, houve uma diferença limítrofe significativa entre as fases ovulatória e folicular do $\mathrm{CM}(\mathrm{p}<0,05)$. No que diz respeito às articulações, não houve diferença significativa entre as fases $(p>0,05)$.

\section{DISCUSSÃO}

O presente estudo procurou analisar o comportamento da flexibilidade durante as diversas fases do CM. Para realizar essa análise consideraram-se universitárias aparentemente saudáveis do ponto de vista de hormônios sexuais e que diferiam apenas quanto à utilização ou não de anovulatórios hormonais. Essa estratégia permitiu a caracterização de níveis hormonais flutuantes ou constantes que se adequaram à necessidade objetiva do estudo, sem precisar modificar a atual rotina das participantes. Não houve nenhum relato de início de gestação ou complicação ginecológica de natureza hormonal no período da coleta dos dados ou no mês seguinte a este. Não houve ainda registro de qualquer intercorrência clínica relevante do sistema locomotor que pudesse ter afetado a realização das medidas passivas de mobilidade articular.

Como sempre ocorre em todo estudo em que não são encontradas evidências de diferenças significativas, algu- mas possibilidades e limitações metodológicas devem ser analisadas. Uma das limitações do nosso estudo, já citada anteriormente, é em relação à definição das fases do CM, que, se fosse medida pelos níveis séricos da razão estrogênio/progesterona na urina ${ }^{15}$ ou no sangue ${ }^{4}$, poderia ter permitido uma discriminação mais precisa da fase do ciclo. Outro aspecto a considerar é a possibilidade de erro na realização da medida. Estudos anteriores sobre o Flexiteste apontam para uma alta fidedignidade intra-avaliador, com uma margem de erro médio entre 1 e 2 pontos ${ }^{14}$. Dessa forma, a diferença encontrada entre medidas consecutivas em nosso estudo - 2 a 4 pontos em média, algo maior para o GE, deve corresponder a diferenças efetivas de flexibilidade e não a um erro de medida. Outro fator que aponta para uma variação real na flexibilidade é o fato de que ela ocorreu mais frequientemente em alguns movimentos, enquanto o erro da medida tende a se distribuir igualmente por todas as 20 medidas. Infelizmente, contudo, não foi possível estabelecer um padrão nítido entre as variações de flexibilidade e as fases do ciclo menstrual. Todavia, podese especular que melhor identificação dos padrões hormonais ou amostra maior ou mais homogênea em relação à flexibilidade ou, ainda, a utilização de métodos de quantificação de lassitude ligamentar mais precisos pudessem ter sido capazes de identificar modificações ainda maiores ou mais expressivas na flexibilidade que não puderam ter sido demonstradas no presente estudo. Existe, assim, necessidade da realização de estudos futuros para esclarecimento mais definitivo dessa questão.

Existe um consenso de que as oscilações hormonais ocorridas nas mulheres devido a alterações, principalmente do estrogênio e progesterona, durante o CM afetam a fisiologia feminina. Em relação ao exercício físico, esse perfil endócrino pode alterar diversos fatores, tais como: a utilização de substrato energético em diferentes intensidades e volumes de treinamento, consumo de oxigênio, armazenamento de substratos energéticos e oxidação de gorduras ${ }^{8}$.

Neste estudo não foram observadas diferenças significativas da flexibilidade, exceto por uma única diferença em um dos índices de variabilidade, o que provavelmente se deveu a um artefato estatístico e não possui maior significado fisiológico. Já Wojtys et $a l^{2}{ }^{2}$, em seu estudo sobre CM e lesão do ligamento cruzado anterior, afirmam que o hormônio relaxina possui habilidade de aumentar a lassitude de tecidos moles. Sua concentração começa a aumentar no $12^{\circ}$ dia, atingindo seu pico no $14^{\circ}$ dia e isso se repete no $20^{\circ}$ dia do ciclo, portanto, no meio da fase lútea, o que nos permite deduzir que, teoricamente, há possibilidades de durante o CM ocorrerem modificações hormonais capazes de causar alterações físicas e metabólicas na expressão da mobilidade articular ou da lassitude ligamentar. É pos- 
sível que as alterações ligamentares específicas induzidas pelas modificações dos níveis circulantes de relaxina não provoquem alterações passíveis de ser identificadas pela análise em escala ordinal limitada em cinco opções do Flexiteste.

Tenaglia et al. ${ }^{6}$ estudaram a influência do CM em mulheres sob o uso de AO e sem o uso do mesmo na tolerância do estresse térmico descompensado. Este estudo encontrou que a temperatura retal se mantém significantemente mais alta no meio da fase lútea comparada com a fase folicular inicial nas mulheres sem o uso de AO e o mesmo ocorre para as mulheres que fazem uso de AO. Os autores sugerem que essas alterações sejam decorrentes do ritmo circadiano e não da administração do hormônio sintético. Esses resultados em parte explicam a ausência de aumento significativo da flexibilidade do nosso estudo; embora a temperatura corporal sofra elevação na fase lútea, é provável que ela não seja suficiente para provocar aumento da flexibilidade nesta fase ou talvez esse aumento não tenha sido detectável.

Em contrapartida, Charkoudian e Johnson ${ }^{7}$ encontraram que a associação de estrogênio e progesterona promove aumento da temperatura corporal de até 0,5 grau centígrado. Em relação ao nosso estudo, os relatos de Charkoudian e Johnson ${ }^{7}$ indicam que no GC os resultados da flexibilidade não deveriam variar, tal como ocorreu, pois estas meninas foram medidas somente durante o período sob o uso de AO e todos os AOs eram compostos de derivados do estrogênio e progesterona, não promovendo, assim, modificação notável nos resultados. Já o GE poderia ter apresentado maior flexibilidade na fase lútea, uma vez que as mulheres foram medidas nas três fases do ciclo.

Outro estudo de Janse de Jonge et al. ${ }^{4}$, que investigou a força de contração máxima isométrica do quadríceps, fadigabilidade e propriedades contráteis estimuladas eletricamente, força de flexão e extensão isocinéticas de joelho e a fadigabilidade e a força de preensão manual durante as fases do CM, mostrou que não houve diferença em nenhuma das funções musculares em uma amostra de 15 mulheres eumenorréicas sem o uso de anovulatório ou qualquer outro hormônio por um período de pelo menos seis meses, sugerindo que as alterações hormonais durante o CM não afetam as variáveis acima. Por outro lado, discordando desses resultados, Sarwar et al. ${ }^{5}$ realizaram um estudo em 20 mulheres, sendo que a metade não usava anovulatório, e compararam os resultados nos dois grupos. Foi encontrado aumento na força do quadríceps e de preensão manual, como também diminuição no tempo de descanso e aumento da fadigabilidade nas mulheres que não faziam uso de anovulatório oral (AO) comparadas com as que usavam. Esses resultados foram encontrados na fase ovulatória. Com isso, os autores propõem que esse aumento da força tenha ocorrido pela alta concentração de estrogênio que antecede a ovulação, sugerindo, dessa forma, que ele aumenta a força muscular, embora, neste estudo as concentrações séricas desse hormônio não tenham sido mensuradas. Em nosso estudo, a determinação da fase do CM foi semelhante à proposta por Sarwar et al. ${ }^{5}$, o que nos leva a inferir a possibilidade de provável limitação para identificarmos os dias do CM.

Em se tratando do treinamento de resistência, as concentrações mais elevadas de estradiol e progesterona têm o poder de alterar a utilização do substrato e a ventilação no repouso e durante o exercício ${ }^{16}$. Bernardes e Radomski ${ }^{17}$ estudaram as alterações nas respostas hormonais do estrogênio e progesterona ao exercício realizado no cicloergômetro, de forma contínua e intermitente, em mulheres jovens que faziam uso de AO. Eles investigaram o efeito dos exercícios em dois momentos: o primeiro foi na fase em que as mulheres não estavam sob o uso de $\mathrm{AO}$ e o segundo, na fase em que elas usavam o AO. A progesterona não respondeu de forma significativa quanto à fase do $\mathrm{CM}$ ou o tipo de exercício. Essa aumentou durante os dois tipos de exercício em todas as condições do ciclo e retornou aos valores de pré-exercício 60 minutos depois de cessado o mesmo ${ }^{17}$.

Em conclusão, as alterações das capacidades físicas apresentadas durante as diferentes fases do CM estão sujeitas a considerável variação individual. Algumas mulheres não apresentam nenhuma alteração perceptível na sua capacidade de desempenho em qualquer período do seu CM, enquanto outras, no entanto, exibem desempenhos variáveis ao longo do ciclo. Estudos futuros utilizando outras populações, amostras maiores e definição hormonal mais precisa das fases do CM são necessários para que essa questão possa ser respondida de forma mais definitiva.

\section{REFERÊNCIAS}

1. Berne RM, Levy MN. Fisiologia. 4a ed. Rio de Janeiro: Guanabara Koogan, 2000;934.

2. Wojtys EM, Huston LJ, Lindenfeld TN, Hewett TE, Greenfield ML. Association between the menstrual cycle and anterior cruciate ligament injuries in female athletes. Am J Sports Med 1998;26:614-9.

3. Vander A, Sherman J, Luciano D. Human physiology - The mechanisms of body function. $8^{\text {th }}$ ed. New York: McGraw-Hill, 2001;654-5.

4. Janse de Jonge XA, Boot CR, Thom JM, Ruell PA, Thompson MW. The influence of menstrual cycle phase on skeletal muscle contractile characteristics in humans. J Physiol 2001;530(Pt 1):161-6.

5. Sarwar R, Niclos BB, Rutherford OM. Changes in muscle strength, relaxation rate and fatigability during the human menstrual cycle. J Physiol 1996;493(Pt 1):267-72 
6. Tenaglia SA, McLellan TM, Klentrou PP. Influence of menstrual cycle and oral contraceptives on tolerance to uncompensable heat stress. Eur J Appl Physiol 1999;80:76-83.

7. Charkoudian N, Johnson JM. Female reproductive hormones and thermoregulatory control of skin blood flow. Exerc Sport Sci Rev 2000;28: 108-12.

8. Braun B, Horton T. Endocrine regulation of exercise substrate utilization in women compared to men. Exerc Sport Sci Rev 2001;29:149-54.

9. Cureton KJ, Collins MA, Hill DW, Mclhanon FM Jr. Muscle hypertrophy in men and women. Med Sports Sci Med 1988;20:338-44.

10. Helge EW, Kanstrup I-L. Bone density in female elite gymnasts: impact of muscle strength and sex hormones. Med Sports Sci Med 2002;34: 174-80.

11. Araújo CGS. Flexiteste - uma nova versão dos mapas de avaliação. Kinesis 1986;2:231-57.
12. Araújo CGS. Medida e avaliação da mobilidade articular: da teoria à prática [Tese de doutorado]. Instituto de Biofísica, UFRJ, 1987.

13. Araújo CGS. Flexitest. Champaign: Human Kinetics [no prelo].

14. Araújo CGS. Flexiteste: proposição de cinco índices de variabilidade da mobilidade articular. Rev Bras Med Esporte 2002;8:13-9.

15. Wilcox AJ, Dunson D, Baird DD. The timing of the "fertile window" in the menstrual cycle: day specific estimates from a prospective study. BMJ 2000;321:1259-62.

16. Tarnalposky LJ, MacDougall JD, Atkinson SA, Tarnalposky MA, Sutton JR. Gender differences in substrate for endurance exercise. J Appl Physiol 1990;68:302-8.

17. Bernardes RP, Radomski MW. Growth hormone responses to continuous and intermittent exercise in females under oral contraceptive therapy. Eur J Appl Physiol 1998;79:24-9. 